\title{
Numerical Aerodynamic Optimization Incorporating Laminar-Turbulent Transition Prediction
}

\author{
J. Driver* and D. W. Zingg \\ University of Toronto, \\ Toronto, Ontario M3H 5T6, Canada
}

DOI: $\underline{10.2514 / 1.23569}$

\begin{abstract}
A two-dimensional Newton-Krylov aerodynamic shape optimization algorithm is applied to several optimization problems in which the location of laminar-turbulent transition is free. The coupled Euler and boundary-layer solver MSES is used to obtain transition locations through the $e^{N}$ method, which are then used in Optima2D, a Newton-Krylov discrete-adjoint optimization algorithm based on the compressible Reynolds-averaged NavierStokes equations. The algorithm is applied to the design of airfoils with maximum lift-to-drag ratio, endurance factor, and lift coefficient. The design examples demonstrate that the optimizer is able to control the transitionpoint locations to provide optimum performance, often producing pressure distributions with laminar rooftops followed by concave pressure recovery. In particular, the optimization algorithm is able to design an airfoil that is very similar, in terms of both shape and performance, to one of the high-lift airfoils designed by Liebeck (Liebeck, R. H., "A Class of Airfoils Designed for High Lift in Incompressible Flow," Journal of Aircraft, Vol. 10, No. 10, 1973, pp. 610-617) in the 1970s. The results provide a striking demonstration of the capability of the NewtonKrylov aerodynamic optimization algorithm to design airfoils with characteristics that previously required a great deal of expertise to achieve.
\end{abstract}

\section{Introduction}

$\mathbf{N}$ UMERICAL optimization techniques based on either gradientbased adjoint methods or genetic algorithms have proven to be a powerful tool in aerodynamic design [1-4]. Most existing optimization algorithms that are based on the Reynolds-averaged Navier-Stokes (RANS) equations assume that the flow is fully turbulent; that is, laminar-turbulent transition is assumed to occur at the leading edge $[2,3,5-7]$. This is primarily because most transitionprediction methodologies are easier to incorporate into the boundarylayer equations and are quite difficult to implement in a NavierStokes framework. As a result, the optimizer cannot exploit the effect of the airfoil shape on the location of transition when maximizing the objective function. Therefore, these algorithms cannot optimize natural-laminar-flow airfoils, in which significant regions of laminar flow are achieved primarily through a suitable pressure gradient.

Before the development of efficient and robust aerodynamic optimization techniques, high-performance airfoils could be designed by determining a pressure distribution that is feasible and optimal in some sense and using an inverse method to find the corresponding airfoil shape. For example, the Stratford concave pressure distribution, which leads to incipient turbulent boundarylayer separation, can be used to produce the shortest region of pressure recovery possible without separation. Liebeck [8] used both laminar and turbulent rooftops followed by Stratford-type pressure recovery to design several high-lift airfoils. Zingg [9] designed an airfoil for a high lift-to-drag ratio by combining a rooftop with a pressure gradient chosen to maintain laminar flow with a concave pressure recovery determined by requiring a constant boundary-layer

Presented as Paper 247 at the 44th Aerospace Sciences Meeting, Reno, NV, 9-12 January 2006; received 1 March 2006; revision received 1 January 2007; accepted for publication 3 May 2007. Copyright @ 2007 by David W. Zingg. Published by the American Institute of Aeronautics and Astronautics, Inc., with permission. Copies of this paper may be made for personal or internal use, on condition that the copier pay the $\$ 10.00$ per-copy fee to the Copyright Clearance Center, Inc., 222 Rosewood Drive, Danvers, MA 01923; include the code 0001-1452/07 \$10.00 in correspondence with the CCC.

*Institute for Aerospace Studies, 4925 Dufferin Street; currently Aeronautical Engineer, Pratt and Whitney Canada; jonathan.driver@ pwc.ca. Member AIAA.

'Professor, Tier I Canada Research Chair in Computational Aerodynamics, Institute for Aerospace Studies, 4925 Dufferin Street. Associate Fellow AIAA. shape factor. The constant shape factor permits a margin from separation to be chosen, leading to rapid pressure recovery without incipient separation. The airfoils designed in these two studies achieve very high performance. Their design is a complex process involving considerable knowledge of aerodynamics. This suggests the following question: Can such airfoils be designed automatically using an aerodynamic optimization technique? There are two aspects to the question. The first issue is whether the optimizer is able to find such a unique and specialized optimum, and the second is whether the airfoils designed by Liebeck []ㅡ and Zingg [9] are actually optimal in some sense; that is, can the optimizer do better?

The objectives of the current work are as follows:

1) We seek to incorporate laminar-turbulent transition prediction into Optima2D, the Newton-Krylov discrete-adjoint aerodynamic optimization algorithm of Nemec and Zingg [2, 3 ], which is based on the Reynolds-averaged Navier-Stokes equations.

2) We seek to investigate whether the resulting optimized airfoils have characteristics comparable to high-performance airfoils designed based on aerodynamic expertise, such as those designed by Liebeck [8] and Zingg [9], which have laminar rooftops and concave pressure recovery.

Our emphasis is on subsonic flows. At this stage, we are not as concerned with the efficiency of the algorithm, nor with the practicality of the resulting airfoils. An efficient discrete-adjoint algorithm incorporating transition prediction requires a prediction methodology that can be effectively integrated into a RANS solver, such as that recently proposed by Langtry and Menter [10]. Furthermore, the airfoils presented here were optimized for performance at a single operating point and consequently are not suitable for practical use, which requires consideration of off-design performance as well. Design of a natural-laminar-flow swept wing also requires consideration of three-dimensional effects, such as crossflow instabilities. Although several researchers (such as Drela [11], Dodbele [12], Green et al. [13], Pralits [14], Kroo and Sturdza [15], and Amoignon et al. [16]) have combined transition prediction with aerodynamic optimization in various ways, the present paper is the first to attempt to use Navier-Stokes-based optimization to design airfoils with specialized pressure distributions (e.g., laminar rooftops and concave pressure recovery) of the type studied here. Such airfoils provide an excellent demonstration of the capabilities of an aerodynamic optimization algorithm. 


\section{Newton-Krylov Approach to Aerodynamic Optimization}

The present algorithm is described in detail in Nemec and Zingg $[2,3]$ and Nemec [17]. The compressible Navier-Stokes equations, together with the turbulence model of Spalart and Allmaras (SA), are solved with a Newton-Krylov method in which the linear system at each Newton iteration is solved using the generalized minimal residual method (GMRES) preconditioned with an incomplete lower-upper (ILU) factorization with limited fill. The gradient is computed using the discrete-adjoint method. The discretized flow and turbulence model equations were differentiated by hand to obtain the Jacobian. The adjoint equation is solved using the same preconditioned Krylov solver used by the flow solver. A new set of design variables is found using a quasi-Newton optimizer in which an estimate of the inverse Hessian based on the Broyden-FannoGoldfarb-Shannon (BFGS) rank-two update formula [18] is used to compute a search direction. If the initial step does not produce sufficient progress toward the minimum, the step size is determined using a cubic line search, which terminates when the strong Wolfe conditions [18] are satisfied. The resulting algorithm provides a highly efficient and reliable approach for aerodynamic design problems governed by the Navier-Stokes equations and has been successfully applied to a wide variety of optimization problems.

The meshes used in the present study have between 14,000 and 25,000 nodes. Using the Newton-Krylov algorithm, a fully converged flow solution on these meshes requires roughly $1 \mathrm{~min}$ on a typical modern single-processor personal computer. The computing time required for a gradient computation is generally less than half of that needed for a converged flow solution. The present flow solutions include physical-model errors caused, for example, by inaccurate prediction of transition points and numerical errors associated primarily with finite mesh densities. The numerical errors associated with the present spatial discretization (second-order centered differences with added numerical dissipation) have been examined through mesh refinement studies [19,20]. Based on these studies, the meshes used here can be expected to produce lift coefficients accurate to within $1 \%$ and drag coefficients accurate to within $5 \%$ for attached and mildly separated flows. The critical factor for successful optimization is not the absolute error, but the accuracy of the relative errors, which is substantially better. Therefore, the present mesh densities are sufficient for meaningful optimization.

The extension of the SA turbulence model to include the laminarturbulent trip functions is fairly straightforward and is discussed in the Newton-Krylov framework by Chisholm and Zingg [21]. To obtain accurate transition locations, a complete solution from MSES [11] is obtained. MSES uses the well-known $e^{N}$ method to predict transition. A value of 9 is used for the critical amplification factor value $N$. The transition locations are then used in the RANS flow solve. This is done to avoid the difficulty of fully coupling an $e^{N}$ method into the RANS solution. For this approach to be effective, the pressure distributions of the MSES and RANS solutions should be similar, which is the case for the airfoils studied here.

\section{Gradient Evaluation}

The gradient is computed using the discrete-adjoint method [2]; thus the cost of computing the gradient is almost independent of the number of design variables. To incorporate the effect of the transition point in the gradient, we modify the discrete-adjoint gradient calculation in the following manner:

$$
\frac{\mathrm{d} \mathcal{J}}{\mathrm{d} \mathcal{X}}=\frac{\partial \mathcal{J}}{\partial \mathcal{X}}-\psi^{T} \frac{\partial \mathcal{R}}{\partial \mathcal{X}}+\frac{\partial \mathcal{J}}{\partial \mathcal{T}} \frac{\partial \mathcal{T}}{\partial \mathcal{X}}
$$

where $\mathcal{R}(\mathcal{X}, Q)$ is the discrete residual function, $\mathcal{J}(\mathcal{X}, Q)$ is the objective function, $Q$ are the conservative flow variables, $\mathcal{X}$ are the design variables, $\mathcal{T}$ are the transition-point locations, and $\psi$ is the adjoint variable. The sensitivity of the objective function with respect to the transition-location movement, $\partial \mathcal{J} / \partial \mathcal{T}$, is combined via the chain rule with the movement of the transition location due to airfoil perturbations, $\partial \mathcal{T} / \partial \mathcal{X}$. This product contains the contribution of the upper and lower transition-location sensitivities, which are denoted by the subscripts up and lo:

$$
\frac{\partial \mathcal{J}}{\partial \mathcal{T}} \frac{\partial \mathcal{T}}{\partial \mathcal{X}}=\frac{\partial \mathcal{J}}{\partial T_{\mathrm{lo}}} \frac{\partial T_{\mathrm{lo}}}{\partial \mathcal{X}}+\frac{\partial \mathcal{J}}{\partial T_{\text {up }}} \frac{\partial T_{\text {up }}}{\partial \mathcal{X}}
$$

A first-order backward finite difference approximation of the $\partial \mathcal{J} / \partial \mathcal{T}$ term is

$$
\begin{aligned}
\frac{\partial \mathcal{J}}{\partial T_{\mathrm{lo}}} & =\frac{\mathcal{J}[X, Q]-\mathcal{J}\left[X, Q\left(X, T_{\mathrm{lo}}-h\right)\right]}{h} \\
\frac{\partial \mathcal{J}}{\partial T_{\text {up }}} & =\frac{\mathcal{J}[X, Q]-\mathcal{J}\left[X, Q\left(X, T_{\text {up }}-h\right)\right]}{h}
\end{aligned}
$$

where $\partial T_{\text {up }} / \partial \mathcal{X}$ and $\partial T_{\mathrm{lo}} / \partial \mathcal{X}$ are found via finite differences of airfoil perturbations using MSES. It is important to note that Eqs. (3) and (4) require a new RANS flow solve for each perturbed state, whereas $\partial T / \partial \mathcal{X}$ requires one MSES flow solve per design variable perturbation. Because we are using MSES to calculate the $\partial \mathcal{T} / \partial \mathcal{X}$ term, it is important to eliminate as much introduced error in the calculation of this term as possible. This is done by reducing the viscous residual in MSES to $10^{-6}$. From numerical experiments, the transition locations converged beyond eight significant digits. Further details regarding the algorithm can be found in Driver [22].

\section{Results and Discussion}

To study the performance of the optimization algorithm, the following objective functions are considered. In the first two, the reciprocal is minimized, and geometric constraints are added to the objective function as penalty terms: 1) maximization of the lift-todrag ratio

$$
\frac{C_{L}}{C_{D}}
$$

2) endurance-factor maximization

$$
\frac{C_{L}^{3 / 2}}{C_{D}}
$$

and 3) maximization of lift coefficient $C_{L}$, with and without some additional penalty terms in the objective function.

\section{A. Lift-to-Drag-Ratio Maximization}

The NACA-0012 airfoil is used as the initial airfoil for the maximization of the lift-to-drag ratio. A single-block, structured Cgrid is used with roughly 18,500 nodes (201 on the airfoil surface). The off-wall spacing is $1 \times 10^{-6}$, the distance to the outer boundary is 24 , and the spacing at the stagnation point and trailing edge is $1 \times 10^{-4}$, for which all distances are expressed in terms of the airfoil chord length. The airfoil is parameterized using $15 \mathrm{~B}$-spline control points, of which 12 are used as design variables; the locations are indicated in Fig. 1. Control points $2-7$ are used as design variables on the lower surface, with control points 9-14 used as design variables

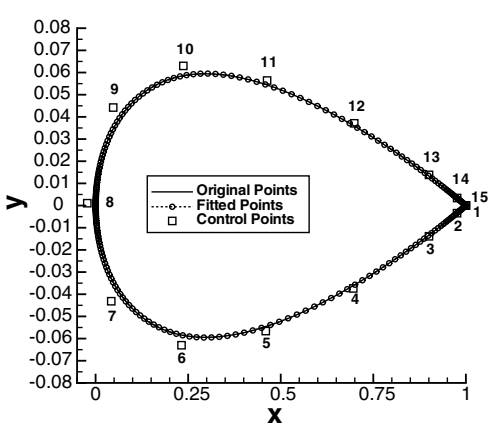

Fig. 1 NACA-0012 control points and design variables. 
Table 1 Lift-to-drag maximization for cases 1 and 2

\begin{tabular}{lrcccc}
\hline \hline \multicolumn{1}{c}{ Case } & $C_{L} / C_{D}$ & $T_{\text {up }}(x / c)$ & $T_{\text {lo }}(x / c)$ & Airfoil area & Angle of attack \\
\hline Case $1_{\text {initial }}$ & 31.38 & 0.45 & 0.85 & 0.08073 & $2.00 \mathrm{deg}$ \\
Case $1_{\text {final }}$ & 101.22 & 0.57 & 0.95 & 0.08081 & $4.23 \mathrm{deg}$ \\
Case $2_{\text {initial }}$ & 28.85 & 0.18 & 0.49 & 0.08073 & $2.00 \mathrm{deg}$ \\
Case $2_{\text {final }}$ & 101.60 & 0.29 & 0.95 & 0.08075 & $5.86 \mathrm{deg}$ \\
\hline \hline
\end{tabular}

on the upper surface. The angle of attack is also included as a design variable. The area of the initial airfoil is required to be preserved throughout the optimization process. The area constraint is lifted into the objective function via a penalty method with a weight of 0.1 .

Two cases are optimized using the preceding initial conditions, but with different operating conditions. Case 1 is optimized at a Mach number of 0.25 and a Reynolds number of $1 \times 10^{6}$, whereas case 2 is optimized at a Mach number of 0.4 and a Reynolds number of $10 \times 10^{6}$. In both cases the initial angle of attack is set to $2 \mathrm{deg}$. Table 1 lists the lift-to-drag ratios, transition locations, and area values for both the initial and final airfoils. Note that the transition points are constrained to lie no further aft than $95 \%$ chord.

Figures 2 and 3 show the initial and final airfoils and pressure distributions. Case 1 shares similar qualities found in high-lift-todrag-ratio airfoils at similar operating conditions [9]. The lower surface experiences laminar flow as far aft as permitted, whereas transition occurs at approximately $57 \%$ chord on the upper surface. Case 2 shows how the Reynolds number affects the optimum, because transition is very sensitive to the Reynolds number. The lower surface again experiences laminar flow to $95 \%$ chord. To maintain a region of laminar flow on the upper surface, the favorable pressure gradient before transition is required to be steeper than in the lower Reynolds number case. This favorable pressure gradient adds stability to the boundary layer, allowing transition to occur further aft.

A lift-to-drag-ratio maximization for Reynolds numbers ranging from 1 to $10 \times 10^{6}$ at a Mach number of 0.25 is presented in Fig. 4 .
Table $\underline{2}$ lists the associated lift-to-drag-ratio values, transition locations, and angles of attack for all of the cases. These results demonstrate how the optimizer modifies the pressure distribution on the upper surface to provide boundary-layer stability at different Reynolds numbers. As the Reynolds number increases, the favorable pressure gradient on the upper surface gradually becomes steeper to maintain boundary-layer stability, as expected.

\section{B. Endurance-Factor Maximization}

The general-aviation airfoil GA(W)-1 is used as the initial airfoil for the endurance-factor maximization. A single-block, structured Cgrid is used with roughly 14,000 nodes (201 on the airfoil surface). The remaining grid details are identical to the preceding lift-to-dragratio maximization case. The airfoil is parameterized using $15 \mathrm{~B}$ spline control points, of which six are used as design variables. The angle of attack is also included as a design variable. Table $\underline{3}$ shows the thickness constraints used to avoid invalid shapes during the optimization iterations. The thickness constraints are lifted into the objective function via a penalty method with a weight of 1.0.

Two cases are optimized using the preceding initial conditions. Case 3 is optimized under fully turbulent conditions at a Mach number of 0.2 and a Reynolds number of $2 \times 10^{6}$. Case 4 is optimized at the same Mach and Reynolds numbers with free transition. Case 3 is the seven-design-variable case presented by Hua et al. [23]. Table 4 lists the endurance factor, transition locations, and angle of attack for both the initial and final airfoils. The initial and

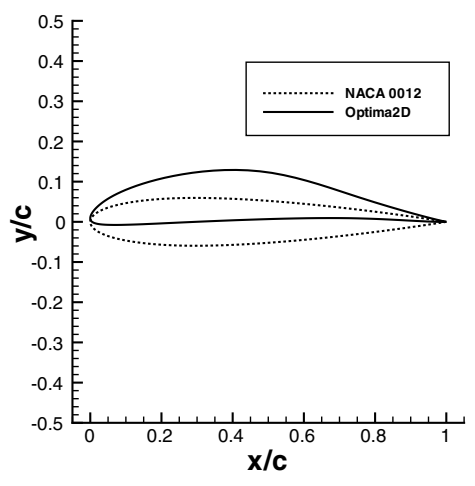

a) Initial and final airfoils

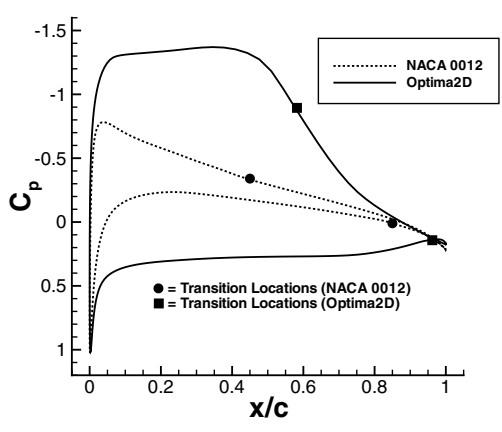

b) Initial and final pressure distributions

Fig. 2 Case 1 lift-to-drag ratio maximization; $M=0.25$ and $R e=1 \times 10^{6}$.

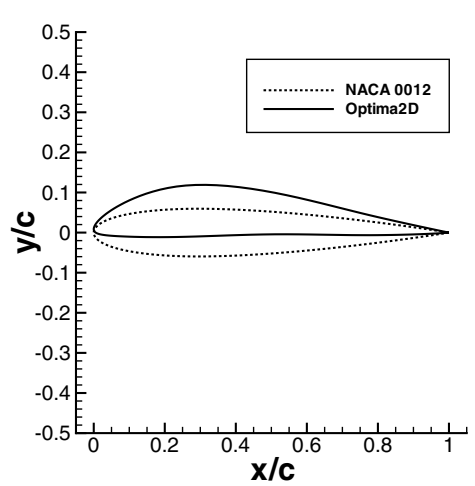

a) Initial and final airfoils

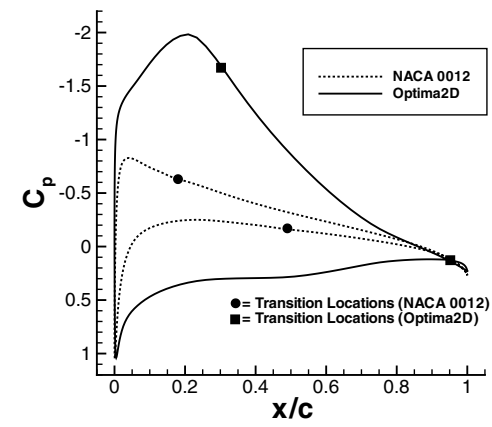

b) Initial and final pressure distributions

Fig. 3 Case 2 lift-to-drag-ratio maximization; $M=0.40$ and $R e=10 \times 10^{6}$. 


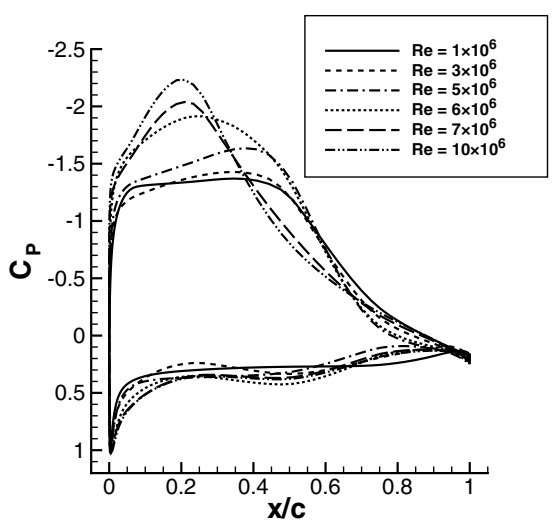

a) Pressure distributions

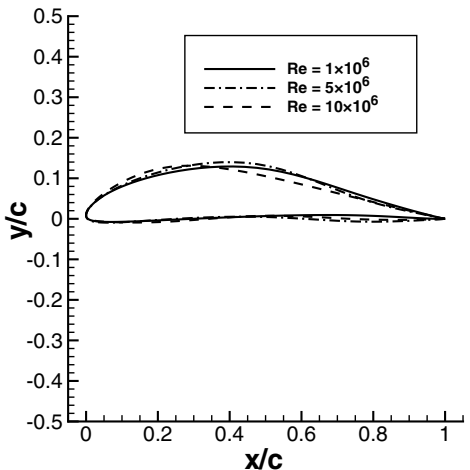

b) Sample airfoils

Fig. 4 Lift-to-drag-ratio maximization; Reynolds number variations.

final airfoils and pressure distributions for case 3 are shown in Fig. $\underline{\text {; }}$; results for case 4 are presented in Fig. 6 . The initial angle-of-attack is chosen to maximize the endurance factor for the $\mathrm{GA}(\mathrm{W})-1$ airfoil under the specified conditions (i.e., fully turbulent or with free transition).

In both cases, the thickness constraint at $35 \%$ chord is the only active constraint at convergence. By exploiting a substantial region of laminar flow on both surfaces, case 4 is able to produce an airfoil that has an endurance factor nearly double that of the fully turbulent case. Although this demonstrates a far superior airfoil at these operating conditions, off-design conditions can have a detrimental impact on the performance. For example, when the airfoil designed with free transition in case 4 is analyzed under fully turbulent conditions, the steep pressure recovery causes turbulent boundarylayer separation, and the drag pays an enormous penalty. Obviously this is not a desirable aerodynamic property. It is desirable to design an airfoil that exploits the advantages of having maximum laminar flow under ideal conditions but also performs well when transition occurs further forward than expected. These tradeoffs can be assembled into a family of noninferior designs, or a Pareto front.

To form a Pareto front, the weighted-sum method is used:

$$
\mathcal{J}=\omega_{\mathrm{ft}} \mathcal{J}_{\mathrm{ft}}+\left(1-\omega_{\mathrm{ft}}\right) \mathcal{J}_{\mathrm{lt}}+\omega_{t} \sum_{j=1}^{N_{c}} C_{j}
$$

Table 2 Lift-to-drag-ratio maximization for Reynolds number variation

\begin{tabular}{ccccc}
\hline \hline $\begin{array}{c}\text { Reynolds } \\
\text { number }\left(10^{6}\right)\end{array}$ & $C_{L} / C_{D}$ & $T_{\text {up }}(x / c)$ & $T_{\mathrm{lo}}(x / c)$ & Angle of attack \\
\hline 1.0 & 101.20 & 0.57 & 0.95 & $4.23 \mathrm{deg}$ \\
3.0 & 118.40 & 0.53 & 0.95 & $4.64 \mathrm{deg}$ \\
5.0 & 117.32 & 0.51 & 0.95 & $5.35 \mathrm{deg}$ \\
6.0 & 112.02 & 0.43 & 0.95 & $6.34 \mathrm{deg}$ \\
7.0 & 102.27 & 0.31 & 0.95 & $6.33 \mathrm{deg}$ \\
10.0 & 100.20 & 0.29 & 0.95 & $6.80 \mathrm{deg}$ \\
\hline \hline
\end{tabular}

Table 3 Endurance-factor maximization for thickness constraints

\begin{tabular}{llllll}
\hline \hline$x / c$ & 0.15 & 0.35 & 0.60 & 0.92 & 0.99 \\
$t / c$ & 0.01 & 0.164 & 0.07 & 0.01 & 0.001 \\
\hline \hline
\end{tabular}

Table 4 Endurance-factor maximization for cases 3 and 4

\begin{tabular}{lrccc}
\hline \hline \multicolumn{1}{c}{ Case } & $C_{L}^{3 / 2} / C_{D}$ & $T_{\text {up }}(x / c)$ & $T_{\text {lo }}(x / c)$ & Angle of attack \\
\hline Case $3_{\text {initial }}$ & 49.24 & - & - & $7.53 \mathrm{deg}$ \\
Case $3_{\text {final }}$ & 59.83 & - & - & $7.24 \mathrm{deg}$ \\
Case 4 initial & 61.99 & 0.49 & 0.65 & $2.44 \mathrm{deg}$ \\
Case $44_{\text {final }}$ & 115.50 & 0.54 & 0.92 & $5.97 \mathrm{deg}$ \\
\hline \hline
\end{tabular}

where $\mathcal{J}_{\mathrm{ft}}$ and $\mathcal{J}_{\text {lt }}$ are the objective function values given by the reciprocal of Eq. (6) under fully turbulent and laminar-turbulent conditions, respectively; $C_{j}$ are geometric constraints; and $\omega_{t}=1.0$. At each Pareto front location a two-point design problem is solved. The two points are an analysis with free transition and a fully turbulent analysis. The initial conditions are identical to cases 3 and 4. The computed Pareto front is shown in Fig. 7, in which the tradeoffs associated with favoring one operating condition over the other are clearly captured. Figure $\underline{8}$ shows selected Pareto front airfoils and the laminar-turbulent pressure distributions. Table 5 lists the coefficients of lift and drag for the selected solutions contained in the Pareto front.

Interesting tradeoffs between fully turbulent and laminarturbulent designs can be understood through this Pareto front. For example, if one is aggressive and uses $\omega_{\mathrm{ft}}=0.1$, then one pays a huge price if transition occurs at the leading edge; the endurance factor drops from approximately 115 to 49 . If one is conservative and uses $\omega_{\mathrm{ft}}=0.9$, then the gain when laminar flow is achieved is minimal. With intermediate values of $\omega_{\mathrm{ft}}$ (for example, $\left.0.3 \leq w_{\mathrm{ft}} \leq 0.7\right)$, the off-design performance improves without too large a penalty in on-design performance. For example, with $\omega_{\mathrm{ft}}=0.5$, the fully turbulent endurance factor is approximately 56 , whereas the on-design laminar-turbulent endurance factor is approximately 107 . It is clear from this Pareto front that the airfoils optimized using $\omega_{\mathrm{ft}}$ values outside the range of $0.3 \leq w_{\mathrm{ft}} \leq 0.7$ are poor choices.

\section{Maximization of Lift Coefficien}

In this section, three different lift-maximization problems are considered. In each case, the NACA-0012 airfoil is the initial airfoil. A single-block, structured C-grid is used with roughly 25,000 nodes (325 on the airfoil surface). The remaining grid details and geometry parameterization are identical to the lift-to-drag-ratio maximization cases. Table 6 shows the thickness constraints used. The thickness constraints are lifted into the objective function via a penalty method with a weight of 0.05 .

For the first lift-maximization example, designated case 5, the following objective function is used [24]:

$$
\mathcal{J}=\omega_{L}\left(1-\frac{C_{L}}{C_{L}^{*}}\right)^{2}+\omega_{t} \sum_{j=1}^{N_{c}} C_{j}
$$

where $C_{L}^{*}$ is a target lift coefficient chosen as 2.10 , and $\omega_{L}=2.0$. The results of this optimization are given in Table 7 and Fig. 9. A lift coefficient of 2.09 is achieved with turbulent boundary-layer separation at $86 \%$ chord on the upper surface. The pressure distribution on the upper surface has a sort of laminar rooftop followed by concave pressure recovery. There is a favorable pressure gradient over the entire lower surface.

For the second lift-maximization example, designated case 6 , a constraint on the skin-friction coefficient is added in the following manner: 


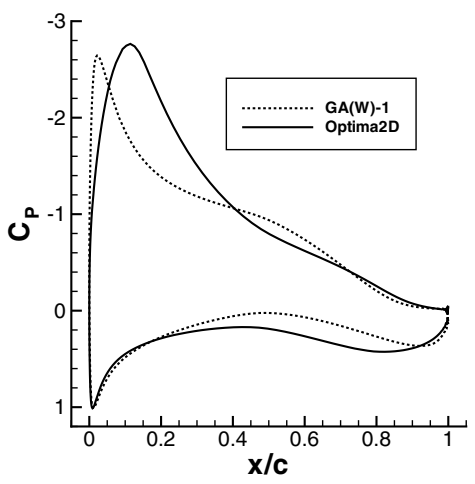

a) Initial and final pressure distributions

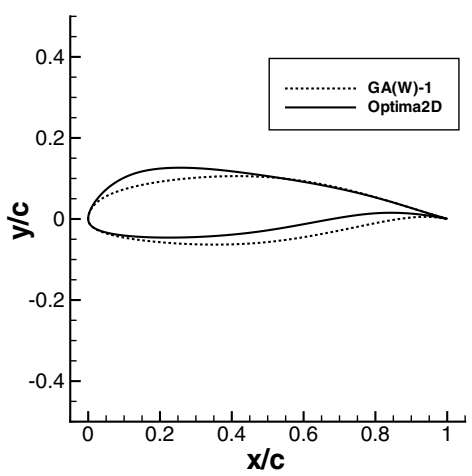

b) Initial and final airfoils

Fig. 5 Case 3 endurance-factor maximization, fully turbulent; $M=0.2$ and $R e=2 \times 10^{6}$.

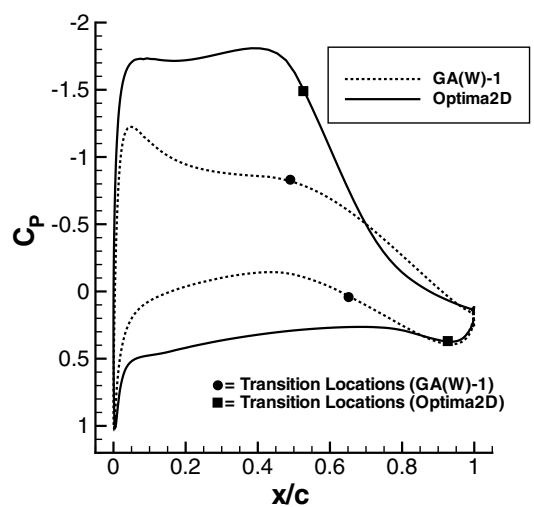

a) Initial and final pressure distributions

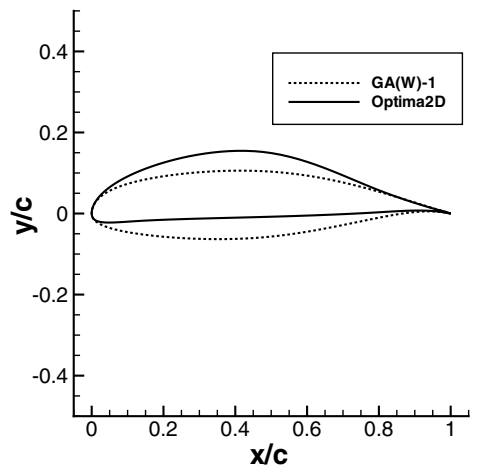

b) Initial and final airfoils

Fig. 6 Case 4 endurance-factor maximization, free transition; $M=0.2$ and $R e=2 \times 10^{6}$.

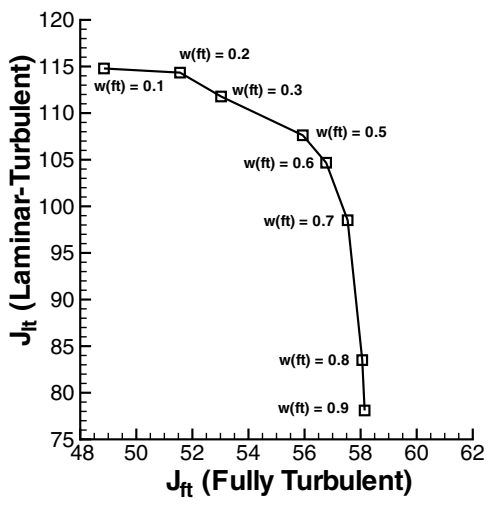

Fig. 7 Pareto front; free transition versus fully turbulent endurancefactor maximization.

$$
\mathcal{J}=\omega_{L}\left(1-\frac{C_{L}}{C_{L}^{*}}\right)^{2}+\sum_{x=x_{\text {start }}}^{x_{\text {end }}}\left[\omega_{c f}\left(1-\frac{c_{f}(x)}{c_{f}^{*}(x)}\right)^{2}\right]+\omega_{t} \sum_{j=1}^{N_{c}} C_{j}
$$

where $c_{f}^{*}$ is a target skin-friction coefficient over a specified range of the airfoil surface from $x_{\text {start }}$ to $x_{\text {end }}$. Note that the skin-friction constraint is active only when violated (i.e., when the local skin friction dips below the specified value). The following parameters are used: $C_{L}^{*}=1.72, c_{f}^{*}=0.001, \omega_{L}=2.0$, and $\omega_{\mathrm{cf}}=1.0$. This constraint ensures that the flow remains fully attached. Results are given in Table $\underline{8}$ and Fig. 10 . With the requirement of fully attached flow, the maximum lift coefficient achieved is 1.69. The uppersurface pressure distribution displays a significant favorable gradient in the laminar region followed by concave pressure recovery. The lower surface again has a favorable pressure gradient throughout.

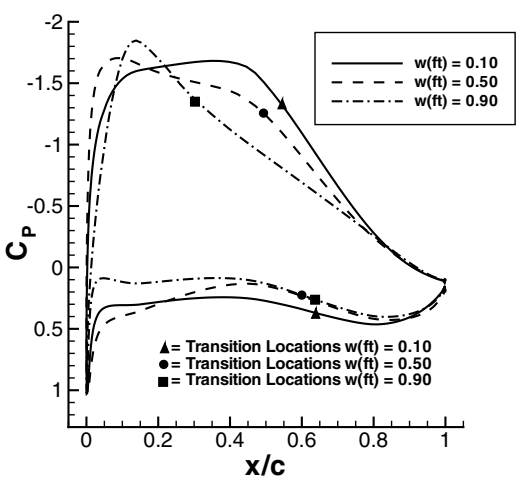

b) Selected Pareto front pressure distributions with free transition

Fig. 8 Selected Pareto-optimal solutions. 
Table 5 Aerodynamic coefficients and endurance factor values for selected Pareto-optimal solutions

\begin{tabular}{ccccccc}
\hline \hline & \multicolumn{2}{c}{ Fully turbulent } & \multicolumn{2}{c}{ Free transition } & Fully turbulent & Free transition \\
$\omega_{\mathrm{ft}}$ & $C_{L}$ & $C_{D}$ & $C_{L}$ & $C_{D}$ & $C_{L}^{3 / 2} / C_{D}$ & $C_{L}^{3 / 2} / C_{D}$ \\
\hline 0.1 & 1.088 & 0.0233 & 1.384 & 0.0142 & 48.84 & 114.7 \\
0.2 & 1.127 & 0.0232 & 1.383 & 0.0142 & 51.55 & 114.2 \\
0.3 & 1.126 & 0.0225 & 1.285 & 0.0130 & 53.02 & 111.7 \\
0.5 & 1.213 & 0.0239 & 1.292 & 0.0137 & 55.90 & 107.5 \\
0.6 & 1.259 & 0.0249 & 1.212 & 0.0128 & 56.72 & 104.4 \\
0.7 & 1.235 & 0.0239 & 1.105 & 0.0118 & 57.48 & 98.34 \\
0.8 & 1.270 & 0.0247 & 1.134 & 0.0145 & 57.96 & 83.31 \\
0.9 & 1.308 & 0.0257 & 1.036 & 0.0135 & 58.01 & 77.86 \\
\hline \hline
\end{tabular}

Table 6 Maximization of lift for thickness constraints

\begin{tabular}{llllll}
\hline \hline$x / c$ & 0.06 & 0.25 & 0.60 & 0.80 & 0.95 \\
$t / c$ & 0.08 & 0.10 & 0.055 & 0.02 & 0.01 \\
\hline \hline
\end{tabular}

Table 7 Maximization of lift for case 5

\begin{tabular}{lccccc}
\hline \hline Case & $C_{L}$ & $C_{D}$ & $T_{\text {up }}(x / c)$ & $T_{\text {lo }}(x / c)$ & Angle of attack \\
\hline Case 5 $5_{\text {initial }}$ & 0.2188 & 0.0066 & 0.37 & 0.75 & $2.00 \mathrm{deg}$ \\
Case $5_{\text {final }}$ & 2.0937 & 0.0277 & 0.36 & 0.95 & $11.70 \mathrm{deg}$ \\
\hline \hline
\end{tabular}

For our third lift-maximization example, case 7, the pitching moment is constrained, and the requirement of attached flow is retained, leading to the following objective function:

$$
\begin{aligned}
\mathcal{J} & =\omega_{L}\left(1-\frac{C_{L}}{C_{L}^{*}}\right)^{2}+\omega_{M}\left(1-\frac{C_{M}}{C_{M}^{*}}\right)^{2} \\
& +\sum_{x=x_{\text {start }}}^{x_{\text {end }}}\left[\omega_{\mathrm{cf}}\left(1-\frac{c_{f}(x)}{c_{f}^{*}(x)}\right)^{2}\right]+\omega_{t} \sum_{j=1}^{N_{c}} C_{j}
\end{aligned}
$$

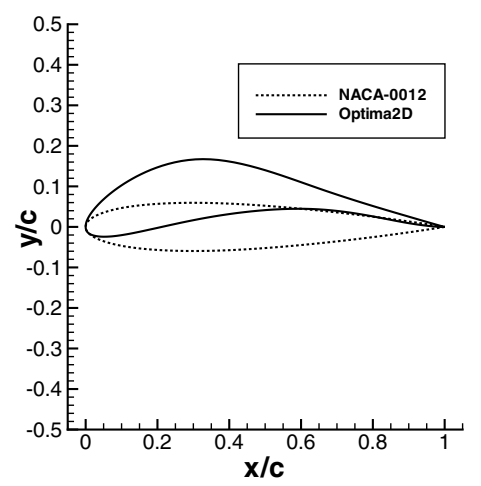

a) Initial and final airfoils
Table 8 Maximization of lift with separation constraint for case 6

\begin{tabular}{cccccc}
\hline \hline Case & $C_{L}$ & $C_{M}$ & $T_{\text {up }}(x / c)$ & $T_{\text {lo }}(x / c)$ & Angle of attack \\
\hline Case $6_{\text {initial }}$ & 0.2188 & 0.0066 & 0.37 & 0.75 & $2.00 \mathrm{deg}$ \\
Case 6 final & 1.6937 & 0.0181 & 0.38 & 0.95 & $8.56 \mathrm{deg}$ \\
\hline \hline
\end{tabular}

The moment constraint is nonzero only if the pitching-moment coefficient exceeds the target value $C_{M}^{*}$ (in magnitude). The following parameters are used: $C_{L}^{*}=1.60, \quad C_{M}^{*}=-0.032$, $c_{f}^{*}=0.001, \omega_{L}=2.0, \omega_{M}=1.0$, and $\omega_{\mathrm{cf}}=1.0$. The optimized airfoil and associated pressure distribution are shown in Fig. 11; further data are given in Table 9. The reduction in the magnitude of the moment coefficient has led to a decrease in the lift coefficient to 1.58. The upper-surface pressure distribution has a laminar rooftop, with a pressure coefficient of roughly -2.50 followed by concave pressure recovery. The lower surface has a more pronounced favorable pressure gradient than the two previous cases, leading to decreased aft loading and consequently a reduced pitching moment. The lower surface of the airfoil is flat, in contrast to the concave lower surfaces of the optimized airfoils of cases 5 and 6 .

Figure 12 compares the lift, drag, and moment coefficients of the optimized airfoils of cases 5, 6, and 7 as a function of angle of attack. In comparison with case 5 , the additional skin-friction coefficient constraint in case 6 has caused a reduction in maximum lift coefficient, a reduction in drag coefficient, and a reduction in the magnitude of the pitching-moment coefficient. The addition of the pitching moment constraint in case 7 has resulted in the same trends. Therefore, of the three optimized airfoils, case 7 has the lowest maximum lift coefficient, the lowest drag coefficient, and the smallest pitching-moment coefficient.

\section{Comparison with Zingg and Liebeck Airfoils}

The previous examples demonstrated that the optimization algorithm is capable of exploiting the location of transition to optimize the airfoil. The resulting pressure distributions correspond

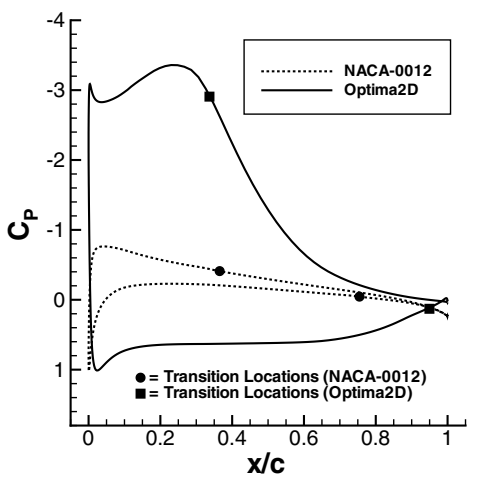

b) Initial and final pressure distributions

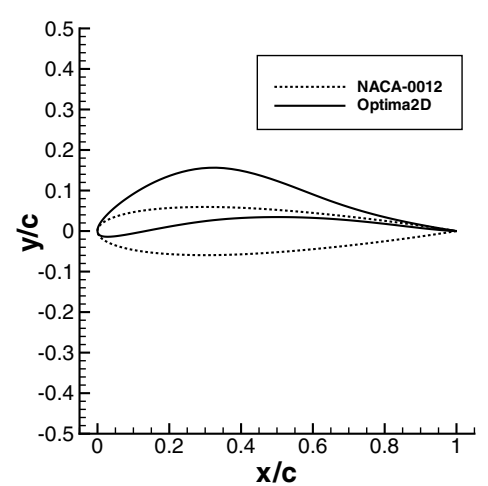

a) Initial and final airfoils

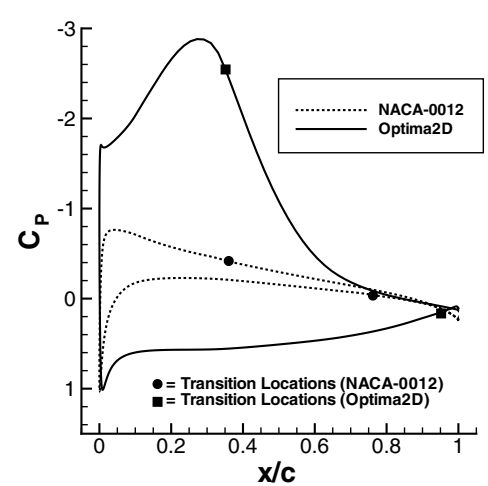

b) Initial and final pressure distributions

Fig. 10 Case 6 maximization of lift with separation constraint; $M=0.25$ and $R e=2 \times 10^{6}$. 


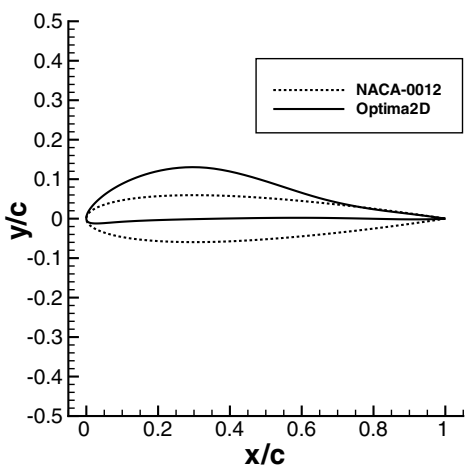

a) Initial and final airfoils

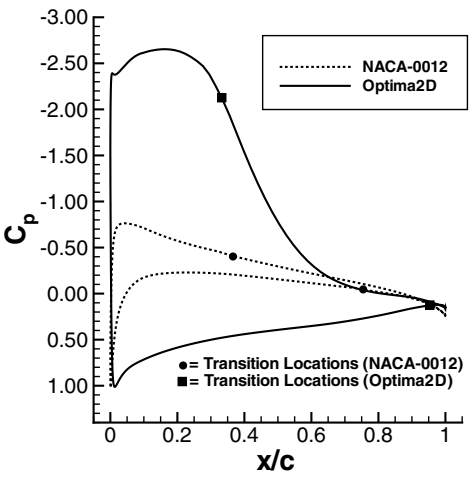

b) Initial and final pressure distributions

Fig. 11 Case 7 maximization of lift with pitching moment and attached-flow constraints; $M=0.25$ and $R e=2 \times 10^{6}$.

Table 9 Maximization of lift with pitching moment and attached constraints for case 7

\begin{tabular}{lcrccc}
\hline \hline Case & $C_{L}$ & \multicolumn{1}{c}{$C_{M}$} & $T_{\text {up }}(x / c)$ & $T_{\text {lo }}(x / c)$ & Angle of attack \\
\hline Case $7_{\text {initial }}$ & 0.2188 & 0.0028 & 0.37 & 0.75 & $2.00 \mathrm{deg}$ \\
Case $7_{\text {final }}$ & 1.5812 & -0.0324 & 0.33 & 0.95 & $10.06 \mathrm{deg}$ \\
\hline \hline
\end{tabular}

closely to what might be expected: some sort of laminar rooftop followed by a concave pressure recovery region. In this section, we compare two optimized airfoils with airfoils designed using a carefully determined pressure distribution and an inverse method: the airfoils of Liebeck [8] and Zingg [9].

The Liebeck [8] airfoil LNV109A was designed to maximize the lift coefficient with fully attached flow and a practical and realistic shape. The objective function and constraints for case 7 were chosen to mimic those of Liebeck. The two airfoils, as well as the pressure distributions at the optimum angle of attack, are compared in Fig. 13. With the exception of a small region near the leading edge, both the airfoils and the pressure distributions are strikingly similar. The lift, drag, and moment coefficients produced by the two airfoils are nearly identical, as shown in Fig. 12.

To compare with the Zing [9] airfoil, we mimic the thickness constraint used in its design by requiring that the maximum thickness be $15 \%$ of the chord, without specifying the chordwise position. Figure 14 displays the resulting airfoils and pressure distributions. They are again very similar, although the Zingg airfoil has a somewhat steeper pressure recovery and transition further aft on the upper surface. The Zingg airfoil has a lift-to-drag ratio of 83 at $C_{L}=0.888$, which occurs at an angle of attack of $1.93 \mathrm{deg}$. The optimized airfoil has a lift-to-drag ratio of 90 and $C_{L}=0.9974$ at an angle of attack of $4.28 \mathrm{deg}$. The optimized airfoil has a larger lift-todrag ratio, but Fig. 14 shows that it has a slightly smaller crosssectional area.

\section{Conclusions}

A Newton-Krylov algorithm for aerodynamic optimization based on the Reynolds-averaged Navier-Stokes equations was augmented to incorporate laminar-turbulent transition and applied to several

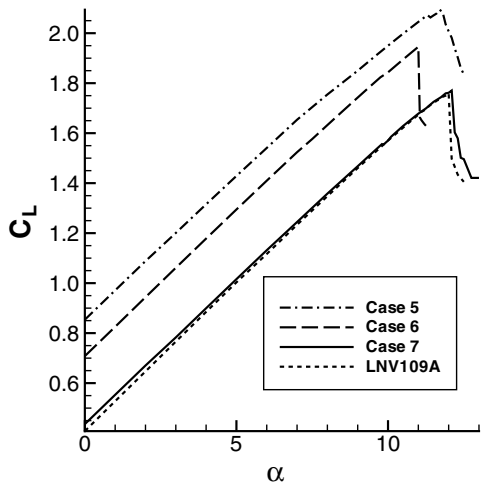

a) $C_{L}$ variation

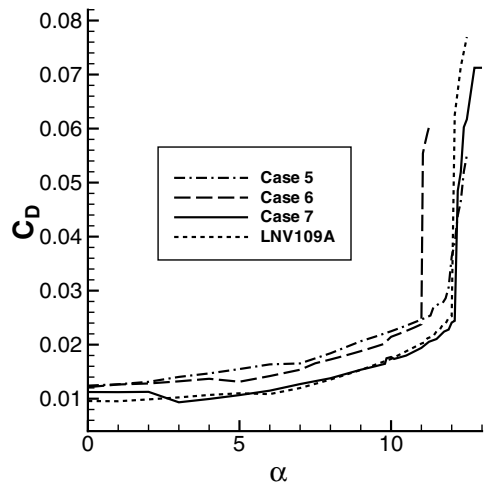

b) $C_{D}$ variation

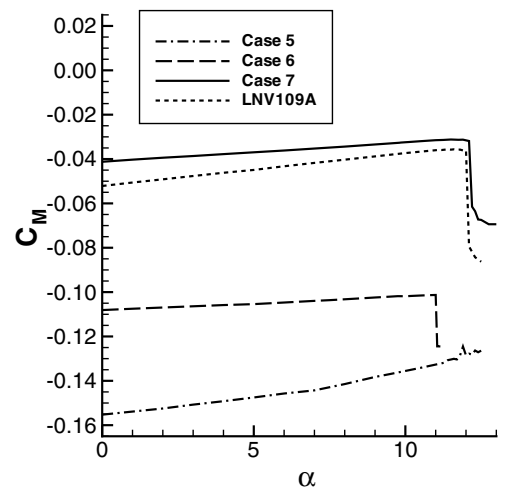

c) $C_{M}$ variation

Fig. 12 Comparison of lift-maximization cases and Liebeck [ $[\underline{8}]$ airfoil (LNV109A); $M=0.25$ and $R e=2 \times 10^{6}$. 


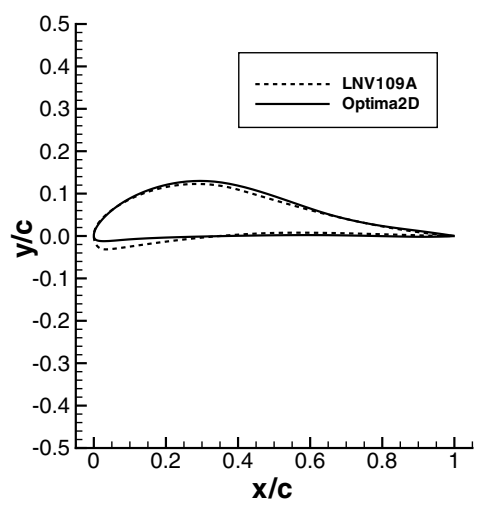

a) Airfoils

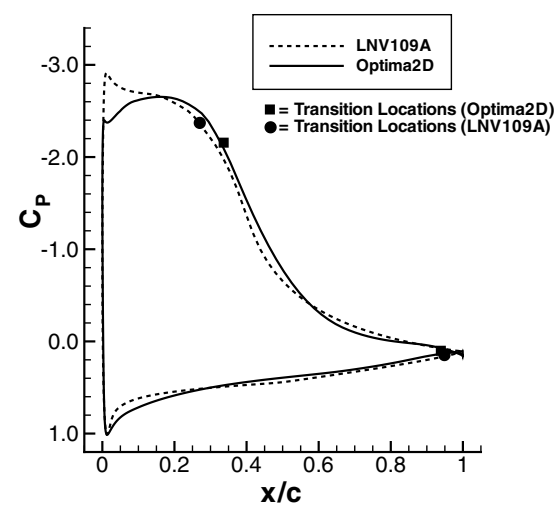

b) Pressure distributions

Fig. 13 Comparison of Liebeck [ $\underline{8}]$ airfoil LNV109A with case 7; $M=0.25$ and $R e=2 \times 10^{6}$.

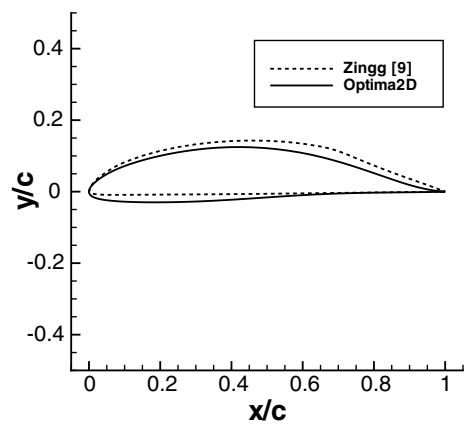

a) Airfoils

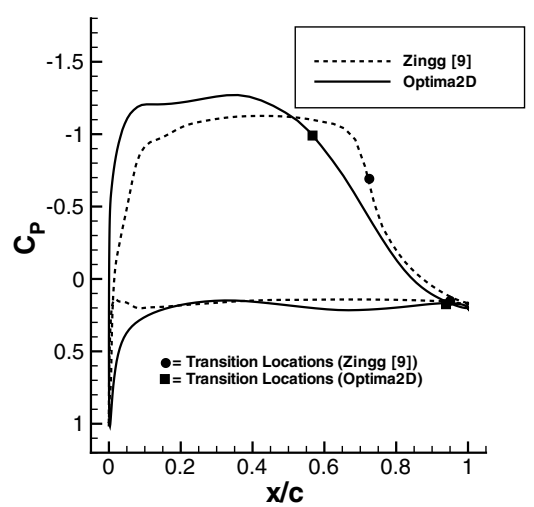

b) Pressure distributions

Fig. 14 Comparison of Zingg [9] airfoil with optimized airfoil; $M=0.25$ and $R e=1 \times 10^{6}$.

aerodynamic optimization problems, including lift-to-drag-ratio maximization, endurance-factor maximization, and lift maximization. The resulting optimized airfoils typically have uppersurface pressure distributions characterized by laminar rooftops followed by concave pressure recovery. Two of the airfoils are remarkably similar to airfoils designed previously by Liebeck [8] and Zingg [9] based on considerable aerodynamic experience. The results provide a striking demonstration of the capability of the Newton-Krylov aerodynamic optimization algorithm to design airfoils that previously required a great deal of expertise to design. Future work will concentrate on integrating the transition-prediction module with the RANS solver to enable full exploitation of the discrete-adjoint method.

\section{References}

[1] Jameson, A., "Aerodynamic Design via Control Theory," NASA CR181749,1988

[2] Nemec, M., and Zingg, D. W., "Newton-Krylov Algorithm for Aerodynamic Design Using the Navier-Stokes Equations," AIAA Journal, Vol. 40, No. 6, 2002, pp. 1146-1154.

[3] Nemec, M., Zingg, D. W., and Pulliam, T. H., "Multipoint and MultiObjective Aerodynamic Shape Optimization," AIAA Journal, Vol. 42, No. 6, 2004, pp. 1057-1065.

[4] Pulliam, T. H., Nemec, M., Holst, T., and Zingg, D. W., "Comparison of Evolutionary (Genetic) Algorithm and Adjoint Methods for MultiObjective Viscous Airfoil Optimizations," AIAA Paper 2003-0298, Jan. 2003.

[5] Jameson, A., Pierce, N. A., and Martinelli, L., "Optimum Aerodynamic Design Using the Navier-Stokes Equations," Theoretical and Computational Fluid Dynamics, Vol. 10, No. 1, 1998, pp. 213-237.

[6] Anderson, W. A., and Bonhaus, D. L., "Airfoil Design on Unstructured Grids for Turbulent Flows," AIAA Journal, Vol. 37, No. 2, 1999, pp. 185-191.
[7] Elliott, J., and Peraire, J., "Progress Toward a Three-Dimensional Aerodynamic Shape Optimization Tool for the Compressible High Reynolds Number Navier-Stokes Equations Discretized on Unstructured Meshes," AIAA Paper 98-2897, June 1998.

[8] Liebeck, R. H., "A Class of Airfoils Designed for High Lift in Incompressible Flow," Journal of Aircraft, Vol. 10, No. 10, 1973, pp. 610-617.

[9] Zingg, D. W., "An Approach to the Design of Airfoils with High Lift to Drag Ratios," M.A.Sc. Thesis, Univ. of Toronto, Toronto, Ontario, Canada, 1981.

[10] Langtry, R. B., and Menter, F. R., "Transition Modeling for General CFD Applications," AIAA Paper 2005-0522, Jan. 2005.

[11] Drela, M., "Design and Optimization Method for Multi-Element Airfoils," AIAA Paper 93-0969, 1993.

[12] Dodbele, S. S., "Design Optimization of Natural Laminar Flow Bodies in Compressible Flow," NASA, TR 4478, 1993.

[13] Green, B. E., Whitesides, J. L., Campbell, R. L., and Mineck, R. E., "A Method for the Constrained Design of Natural Laminar Flow Airfoils," AIAA Paper 96-2502, 1996.

[14] Pralits, J., "Optimal Design of Natural and Hyrbid Laminar Flow Control on Wings," Ph.D. Thesis, Department of Mechanics, Royal Inst. of Technology, Stockholm, Sweden, Oct. 2003.

[15] Kroo, I., and Sturdza, P., "Design-Oriented Aerodynamic Analysis for Supersonic Laminar Flow Wings," AIAA Paper 2003-0774, 2003.

[16] Amoignon, O., Pralits, J., Hanifi, A., Berggren, M., and Henningson, D., "Shape Optimization for the Delay of Laminar-Turbulent Transition," AIAA Journal, Vol. 44, No. 5, 2006, pp. 1009-1024.

[17] Nemec, M., "Optimal Shape Design of Aerodynamic Configurations:A Newton-Krylov Approach," Ph.D. Thesis, Univ. of Toronto, Toronto, Ontario, Canada, 2003.

[18] Nocedal, J., and Wright, S. J., Numerical Optimization, SpringerVerlag, New York, 1999.

[19] Zingg, D. W., "Grid Studies for Thin-Layer-Navier-Stokes Computations of Airfoil Flowfields," AIAA Journal, Vol. 30, No. 10, 1992, pp. 2561-2564.

[20] Zingg, D. W., De Rango, S., Nemec, M., and Pulliam, T. H., "Comparison of Several Spatial Discretizations for the Navier-Stokes 
Equations," Journal of Computational Physics, Vol. 160, No. 2, 2000, pp. 683-704.

[21] Chisholm, T., and Zingg, D. W., "A Fully Coupled Newton-Krylov Solver for Turbulent Aerodynamic Flows," International Council of the Aeronautical Sciences, Paper 2002-333, Toronto, Ontario, Canada, Sept. 2002.

[22] Driver, J., "Optimal Aerodynamic Shape Design with Transition Prediction," M.A.Sc. Thesis, Univ. of Toronto, Toronto, Ontario, Canada, 2005.
[23] Hua, J., Liu, P. J., and Zingg, D. W., "Optimization of Long-Endurance Airfoils," AIAA Paper 2003-3500, June 2003.

[24] Nemec, M., and Zingg, D. W., "Optimization of High-Lif Configurations Using a Newton-Krylov Algorithm," AIAA Paper 2003-3957, 2003.

X. Zhong Associate Editor 